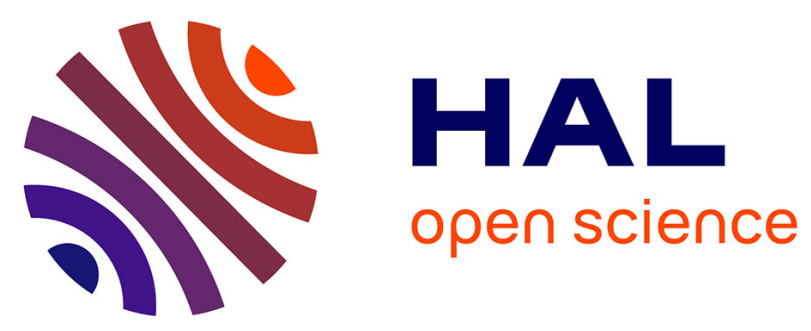

\title{
Cytostatic effect of repeated exposure to simvastatin, a mechanism for chronic myotoxicity revealed by the use of mesodermal progenitors derived from human pluripotent stem cells.
}

Delphine Peric, Isabel Barragan, Karine Giraud-Triboult, Anne-Laure Egesipe, Laurène Meyniel-Schicklin, Christelle Cousin, Vincent Lotteau, Vincent Petit, Jawida Touhami, Jean-Luc Battini, et al.

\section{- To cite this version:}

Delphine Peric, Isabel Barragan, Karine Giraud-Triboult, Anne-Laure Egesipe, Laurène MeynielSchicklin, et al.. Cytostatic effect of repeated exposure to simvastatin, a mechanism for chronic myotoxicity revealed by the use of mesodermal progenitors derived from human pluripotent stem cells.. STEM CELLS, 2015, 33, pp.2936-2948. 10.1002/stem.2107 . hal-01216232

\author{
HAL Id: hal-01216232 \\ https://hal.science/hal-01216232
}

Submitted on 20 Oct 2015

HAL is a multi-disciplinary open access archive for the deposit and dissemination of scientific research documents, whether they are published or not. The documents may come from teaching and research institutions in France or abroad, or from public or private research centers.
L'archive ouverte pluridisciplinaire HAL, est destinée au dépôt et à la diffusion de documents scientifiques de niveau recherche, publiés ou non, émanant des établissements d'enseignement et de recherche français ou étrangers, des laboratoires publics ou privés. 
Running Title: Simvastatin repeated-dose toxicity in vitro

Cytostatic effect of repeated exposure to simvastatin, a mechanism for chronic myotoxicity revealed by the use of mesodermal progenitors derived from human pluripotent stem cells.

Delphine Peric ${ }^{\mathrm{a}, \mathrm{b}, \#}$, Isabel Barragan ${ }^{\mathrm{c}, \#}$, Karine Giraud-Triboult ${ }^{\mathrm{d}}$, Anne-Laure Egesipe ${ }^{\mathrm{a}, \mathrm{b}}$, Laurène Meyniel-Schicklin ${ }^{\mathrm{e}, \mathrm{f}}$, Christelle Cousin ${ }^{\mathrm{g}}$, Vincent Lotteau ${ }^{\mathrm{e}, \mathrm{f}}$, Vincent Petit $^{\mathrm{g}}$, Jawida Touhami $^{\text {h }}$, Jean-Luc Battini ${ }^{\mathrm{h}}$, Marc Sitbon ${ }^{\mathrm{h}}$, Christian Pinset ${ }^{\mathrm{a}, \mathrm{b}}$, Magnus Ingelman-Sundberg ${ }^{\mathrm{c}}$, Delphine Laustriat ${ }^{\mathrm{d}}$, Marc Peschanski ${ }^{\mathrm{a}}{ }^{\mathrm{b}} *$

a. INSERM U861, I-Stem, Evry 91030 cedex France

b. UEVE U861, I-Stem, Evry 91030 cedex France

c. Karolinska Institute, Department of Physiology and Pharmacology, 17177 Stockholm Sweden

d. CECS/AFM, I-Stem, Evry 91030 cedex France

e. CIRI, International Center for Infectiology Research, Université de Lyon, 69007 Lyon, France

f. INSERM U1111, 69007 Lyon, France

g. METAFORA biosystems, Evry 91000 France

h. Institut de Génétique Moléculaire de Montpellier, CNRS, UMR5535, Université de Montpellier, Montpellier 34293, France

\# These authors contributed equally to this work.

*Corresponding author: Marc Peschanski, MD, PhD, I-Stem, Genopole campus 1, 5 rue Henri Desbruères Evry 91030 cedex France, Tel: +33169908517, Fax: +33169908521, Email: $\underline{\text { mpeschanski@istem.fr }}$ 
Author contributions: DP: conception and design, collection and assembly of the data, data analysis and interpretation, manuscript writing. IB: collection and assembly of the data, data analysis and interpretation, manuscript writing. KGT, ALE: collection and assembly of the data, data analysis and interpretation. LMS, VL, CP, MIS: data analysis and interpretation. CC: collection and assembly of data. VP: collection and assembly of the data, data analysis and interpretation, expertise regarding RBD. JT, JLB, and MS: expertise regarding RBD. DL: conception and design, collection and assembly of the data, data analysis and interpretation. MP: conception and design, data analysis and interpretation, manuscript writing. All authors critically reviewed the manuscript.

Keywords: chronic toxicity, pluripotent stem cells, mesoderm, simvastatin, cytostatic agent. 


\begin{abstract}
Statin treatment of hypercholesterolemia can lead to chronic myotoxicity which is, in most cases, alleviated by drug withdrawal. Cellular and molecular mechanisms of this adverse effect have been elusive, in particular because of the lack of in vitro models suitable for longterm exposures. We have taken advantage of the properties of human pluripotent stem cellderived mesodermal precursors, that can be maintained unaltered in vitro for a long period of time, to develop a model of repeated exposures to simvastatin during more than two weeks. This approach unveiled major differences, both in functional and molecular terms, in response to single versus repeated-dose exposures to simvastatin. The main functional effect of the in vitro simvastatin-induced long-term toxicity was a loss of proliferative capacity in the absence of concomitant cell death, revealing that cytostatic effect could be a major contributor to statin-induced myotoxicity. Comparative analysis of molecular modifications induced by simvastatin short-term versus prolonged exposures demonstrated powerful adaptive cell responses, as illustrated by the dramatic decrease in the number of differentially expressed genes, distinct biological pathway enrichments, and distinct patterns of nutrient transporters expressed at the cell surface. This study underlines the potential of derivatives of human pluripotent stem cells for developing new approaches in toxicology, in particular for chronic toxicity testing.
\end{abstract}




\section{Introduction}

The precise mechanisms of statin-induced myotoxicity, a common adverse effect of chronic treatment with those worldwide prescribed anti-cholesterol drugs, have remained elusive. For two decades, statins have been prescribed for preventing cardiovascular diseases through lowering elevated plasmatic concentrations of low-density lipoprotein cholesterol (LDL-C) [1]. These drugs target the enzyme HMG-CoA reductase (HMGCR), thus inhibiting the production of mevalonate, a critical intermediary in the cholesterol biosynthesis pathway [2]. Although statins are well tolerated by most patients, their use has been associated with myotoxicity that translates into myalgia in up to $5 \%$ of the patients, and in rare occasions into more serious cases of rhabdomyolysis [3]. As a consequence, cerivastatin was withdrawn from the market in 2001 after being associated with 52 fatal cases of rhabdomyolysis [4]. More recently, the US Food and Drug Administration has also restricted the use of simvastatin because of an association of the highest approved dose with an elevated risk of muscle injury (http://www.fda.gov/Drugs/DrugSafety/ucm256581.htm).

Despite a wealth of studies, the precise mechanisms of those statin-associated muscle injuries remain controversial. While at the molecular level the depletion of several intermediates of the HMGCR pathway downstream of mevalonate has been incriminated [5], the precise functional consequences are still debated. Primary cultures originating from human biopsies $[6-8]$, mouse $[9,10]$ or rat $[11,12]$ muscle tissues, as well as myotubes derived from immortalized myoblast cell lines [9, 13-15] have been used to study statin-induced myotoxicity in vitro. These experiments have pointed to cell death, either of post-mitotic differentiated myotubes and myofibers following vacuolization $[7,9,11,12,14]$, or of proliferating myoblasts, possibly through apoptosis [13], or of both cell types $[6,16]$.

One particular drawback of the current in vitro cell models is the difficulty in maintaining the cells unaltered for long periods of time, while the statin-induced myotoxicity is essentially a 
consequence of chronic exposure. Most in vitro studies have, thus, focused on single dose exposures, with short-term follow up for $48 \mathrm{~h}$ to $72 \mathrm{~h}$. To our knowledge, no in vitro study beyond 5 days of treatment has been reported so far $[9,12,16]$. It is therefore paradoxical that only acute exposures to statins have been used to evaluate mechanisms for long-term effects on muscle cells.

In this study, we have exploited the advantages of human embryonic stem cell (hES)-derived mesodermal precursors, upstream of the myogenic lineage [17], that can be maintained unaltered in vitro for a long period of time, to explore simvastatin toxicity in an experimental setup allowing a more protracted follow up. We report major differences, both in functional and molecular terms, in response to single versus repeated-dose exposures to simvastatin, pointing to a cytostatic effect, with suggestion of a phenotypic change and metabolic reprogramming, as a primary mechanism for chronic myotoxicity. 


\section{Material and Methods}

hES cell culture. The hES cell line SA01 (Cellartis) was grown on a layer of mouse embryonic fibroblasts (Globalstem) mitotically inactivated with $10 \mu \mathrm{g} / \mathrm{ml}$ mitomycin C. Cultures were carried in Knock-Out (KO)-DMEM supplemented with $20 \%$ KO Serum Replacement (KSR), $2 \mathrm{mM}$ glutamax, $1 \%$ non essential aminoacids, $50 \mu \mathrm{M} \quad \beta$ mercaptoethanol and $10 \mathrm{ng} / \mathrm{mL}$ recombinant human fibroblast growth factor 2 (FGF2) (all from Life technologies). Medium was changed daily and hES cells were manually passaged every 5-7 days.

Differentiation of hES cells into MPCs. Mesodermal precursor cells (MPCs) were obtained by differentiation of SA01 hES cells using an adaptation of a previously described protocol [18]. Briefly, differentiation was induced by plating $4-6 \mathrm{hES}$ colonies $/ \mathrm{cm}^{2}$ on $0.1 \%$ gelatincoated dishes in the presence of KO-DMEM supplemented with $20 \%$ fetal bovine serum (FBS) (Hyclone), $2 \mathrm{mM}$ glutamax, $1 \%$ nonessential aminoacids, $50 \mu \mathrm{M} \beta$-mercaptoethanol, $10 \mathrm{ng} / \mathrm{mL}$ recombinant human FGF2 (all from Life technologies) and $1 \mathrm{mM}$ L-Ascorbic acid 2-phosphate (Aa2-P) (Sigma-Aldrich). Medium was changed every other day and cells were passaged with TrypLE Express (Life technologies) to new gelatin-coated dishes when they reached confluence. MPCs were frozen after approximately 15 days of differentiation (passage 3). For all subsequent experiments, MPCs were thawed from the same differentiated population and cultured on $0.1 \%$ gelatin-coated dishes in a medium similar to that used for differentiation from hES but without FGF2 and Aa2-P.

\section{Production of RBD soluble ligands}


IgG-Fc tagged RBD fusion proteins were produced as described previously [19]. HERVW.RBD.mFc and PERVB.RBD.mFc ligands were derived from the sequences encoding the first 121 and 238 amino acids of the HERVW and PERVB Env respectively. The corresponding RBD-encoding sequence was fused to the mouse IgG1-Fc fragment ( $\mathrm{mFc}$ ). Samples were aliquoted and stored at $-80^{\circ} \mathrm{C}$. Each preparation was verified for integrity by western blotting. Details on produced RBDs are summarized in Table S3.

Dose response experiments of the toxicity of statins. Statins were purchased from Tocris with the exception of cerivastatin, which was purchased from Santa Cruz Biotechnology. Primary myoblasts, hES-derived MPCs and hES-derived NSCs were seeded in black/clear bottom 384-well plates at a density of 1,500 cells/well for myoblasts and MPCs and 3000 cells/well for NSCs. Myotubes in 384-well plates were obtained and characterized as described in Supplementary Methods. $24 \mathrm{~h}$ after seeding or 6 days after beginning differentiation for myotubes, cells were treated with statins at concentrations ranging from $0.0025 \mu \mathrm{M}$ to $16.7 \mu \mathrm{M}$ during $48 \mathrm{~h}$. Vehicle DMSO was used as a control treatment. For MPCs, experiment was performed in parallel with or without the addition of $200 \mu \mathrm{M}$ mevalonate. Each treatment was done in quadruplicate in the plates. Seeding and treatments were performed on an automated liquid handling platform (Bravo, Agilent). After $48 \mathrm{~h}$ of treatment, dead cells and debris were eliminated by washing cells with PBS, remaining cells were fixed with $4 \%$ paraformaldehyde in PBS and nuclei were stained with Hoechst (Life technologies). Cell nuclei were automatically quantified using the LEAP platform (Cyntellect) or the Arrayscan high content imaging reader (Cellomics). Dose response curves, EC50 and $\mathrm{E}_{\max }$ values were calculated using the variable slope model for nonlinear regression from the Prism (Graphpad software). 
Repeated dose treatments with simvastatin. MPCs were seeded in $0.1 \%$ gelatin-coated tissue culture flasks at a low density of 3,125 cells $/ \mathrm{cm}^{2}$ and treated with $0.4 \mu \mathrm{M}, 1 \mu \mathrm{M}$ or 2.5 $\mu \mathrm{M}$ simvastatin or with DMSO adjusted to a final concentration equivalent to the $1 \mu \mathrm{M}$ simvastatin dose. Media and treatments were renewed every other day during 16 days. MPCs were passaged with TrypLE Express (Life technologies) and counted on an automated cell counter (Countess ${ }^{\circledR}$, Life technologies) every 7 days (when they reached $90 \%$ confluence). When passaged, treated MPCs were plated back to 3,125 cells $/ \mathrm{cm}^{2}$. The dilution was taken into account for the calculation of cumulated cell number. Only cells treated with the $2.5 \mu \mathrm{M}$ dose were not passaged and were counted on day 3 and 7. Mortality and proliferation of MPCs were quantified by flow cytometry 2 days post-passage, i.e. at day 2, day 9 and day 16 . For this purpose, MPCs were plated at a density of 8,000 cells $/ \mathrm{cm}^{2}$.

Flow cytometry analyses. Mortality. MPCs were labeled with FITC AnnexinV and propidium iodide (PI) from the FITC AnnexinV Apoptosis Detection Kit I (BD Pharmingen) following the manufacturer's instructions. Assay and gating have been set up with nonlabeled MPCs and non-treated labeled MPCs as negative controls, and MPCs treated $12 \mathrm{~h}$ with staurosporine as AnnexinV+/PI- early apoptotic positive control. Cells were analyzed on the MACSQuant flow cytometer (Miltenyi Biotec). 30,000 events were recorded for each sample analyzed. Dead cells were quantified as AnnexinV+/PI+ and early apoptotic cells were quantified as Annexin V+/PI- using FlowJo software (Tree Star Inc.). Proliferation. Proliferation rate was measured using the Click-iT EdU Alexa Fluor 647 Flow Cytometry Assay Kit (Life technologies) according to the manufacturer's protocol except that fixation was performed using $70 \%$ ethanol in PBS. $10 \mu \mathrm{M}$ Edu was added to the culture medium during $2 \mathrm{~h}$ before harvesting and fixing the cells. After the Click-iT reaction, PI (SigmaAldrich) was used to stain DNA and fluorescence signals were recorded on the MACSQuant 
flow cytometer (Miltenyi Biotec). 30,000 events were recorded for each sample analyzed. Edu content, i.e. cells in S phase of the cell cycle, was quantified using FlowJo software (Tree Star Inc.) by plotting Edu fluorescence against DNA content fluorescence in the proliferating subpopulation, characterized by a small size and by Edu incorporation. RBD Binding to Nutrient Transporters. MPCs were detached using TrypLE Express (Life technologies) for 5 minutes at $37^{\circ} \mathrm{C}$ and transferred into a 96-well $\mathrm{V}$-shape microplate with $3.10^{4}$ cells used for each binding set. RBD were premixed pairwise (one mouse Fc-fused and one rabbit Fc-fused RBD) in culture medium containing $0.1 \%$ sodium azide and $1 \mathrm{mM}$ EDTA. RBD were added to MPCs and incubated at $37^{\circ} \mathrm{C}$ for $20 \mathrm{~min}$. Cells were washed once with PBS/2\% FCS and then incubated with Alexa Fluor 647 goat anti-rabbit IgG (Life technologies, 1:400) and R-PE goat anti-mouse IgG1 (Life technologies, 1:200) antibodies in binding buffer (PBS/2\% FCS/0.1\% sodium azide/1mM EDTA), containing $0.3 \mu \mathrm{g} / \mathrm{mL}$ DAPI to restrict the analysis to live cells. After 30 min of incubation at $4^{\circ} \mathrm{C}$, cells were washed and resuspended in binding buffer before flow cytometry analysis. Fluorescent signals were acquired on a FACSVerse flow cytometer (BD Biosciences) with 405, 488 and $640 \mathrm{~nm}$ excitation, and data analysis was performed using Flowjo software (Tree Star Inc.). Dead cells were excluded from the analysis. "Fluorescence minus one" (FMO) controls were used to establish background levels in RBD channels (R-PE and AF647). Signals were converted into molecules of equivalent soluble fluorochrome (MESF) values using calibration beads (R-PE and AF647 MESF Quantum Beads; Bangs Laboratories) according to the manufacturer's instructions. Hierarchical clustering of RBD binding data was performed with dChip software (Harvard).

Whole genome transcriptomic analyses. Whole-genome transcriptomic analyses of MPCs treated for 2 days or 17 days with simvastatin $1 \mu \mathrm{M}$ and of the corresponding DMSO-treated 
controls were performed in triplicate. Total RNA was isolated using the RNeasy Mini kit (Qiagen) with DNAse I digestion. RNA concentration and quality were measured using the NanoDrop ND-1000A spectrophotometer. RNA integrity was evaluated on Agilent Bioanalyzer 2100. Using 200 ng of RNA as input material, sense strand cDNA was generated with Ambion WT expression kit (PN 900671). The Affymetrix GeneChip WT Labeling Kit (PN 900671) was then used to fragment and biotin label sense target for hybridization onto Affymetrix Human Gene 1.1 ST array plates containing 36,079 RefSeq transcripts. Hybridization Cocktail was prepared according to Affymetrix standard protocol for Gene 1.1 STArray plates. The Gene Titan Instrument system was used for hybridization, washing, staining and scanning. Gene array processing was performed at the Bioinformatics and Expression Analysis (BEA) core facility, Karolinska Institutet (Stockholm). Gene expression data and experimental information is available at EBI ArrayExpress (https://www.ebi.ac.uk/arrayexpress) under accession number E-MTAB-2753. Data analysis of the Affymetrix array plates results was performed with Affymetrix Expression Console (EC, version 1.1) using the following methods: (i) summarization: PLIER, (ii) background correction: PM-GCBG and (iii) normalization: Global Median. Expression levels in the treatment groups were compared to the control groups using Student's t-test (2-sided, unpaired) analysis. For each time point, a list of differentially expressed (DE) genes was generated, using 2 and 0.05 as fold change and p-value cut-off values, respectively. The Database for Annotation, Visualization and Integrated Discovery (DAVID) was used for biological processes enrichment analysis. Ingenuity Pathway Analysis v.9 (IPA) (Ingenuity Systems, Redwood, CA, USA) was used as a complement to identify Physiological System Development and Function terms modulated by the simvastatin exposure. 
Quantitative RT-PCR. Total RNA was isolated using the RNeasy Mini kit (Qiagen) with DNAse I digestion. The RNA samples were quantified and assessed for integrity using the NanoDrop ND-1000A spectrophotometer or the Agilent Bioanalyzer 2100 with the RNA 6000 Nano kit (Agilent Technologies). Reverse transcription was performed with SuperScript III reverse transcriptase and random primers (Life Technologies). Gene expression was determined by quantitative PCR performed with SYBR Green PCR Master Mix or TaqMan® Probe-Based Gene Expression Analysis (Life Technologies) on the 7900HT Fast Real-Time PCR System (Life Technologies). Primer sequences and TaqMan ${ }^{\circledR}$ assays information are presented in Table S4. Quantification of gene expression was based on the deltaCt method and normalized to beta-tubulin expression.

Statistical analysis. Statistical analysis were performed using either one way analysis of variance (ANOVA) followed by a Dunnett's multiple comparison test or using one sample t test when relative data normalized to DMSO treated samples are presented. P-values $<0.05$ were considered significant. 


\section{Results}

\section{Dose-response curves of statin-induced acute toxicity on MPCs.}

hES-derived mesodermal precursor cells (MPCs) were obtained as an homogeneous population after 16 days of differentiation and maintained a normal karyotype in culture (Fig. S1A-B). They expressed homogeneously the mesenchymal surface markers CD29, CD166, CD105, CD73 and CD44 and were negative for the pluripotent marker Tra-1-81 (Fig. S1C). At least 50\% of MPCs expressed alpha-smooth muscle actin ( $\alpha$-SMA) (Fig. S1D).

The sensitivity of MPCs to statin toxicity was evaluated by a single dose exposure of $48 \mathrm{~h}$ to increasing concentrations of 6 statins and the quantification of residual cell numbers (Fig. 1A). Cerivastatin was by far the most toxic compound tested with an efficient concentration for a $50 \%$ reduction of the cell number (EC50) of $0.07 \mu \mathrm{M}$ (Table 1). At the other end of the spectrum, pravastatin was not toxic at any concentration tested, as previously described in other in vitro systems and associated to its hydrophilic properties that may prevent passive entry into cells $[11,16,20]$. All other statins displayed similar dose-response curves, with EC50s in the micromolar range. Complete rescue of the toxic effects by addition of mevalonate showed that statin-induced toxicity was specifically due to HMGCR inhibition in MPCs (Fig. 1B).

Dose-response curves for statin toxicity were established with three additional cell types for comparison purposes. First, statins were tested on primary human muscle cells at two stages of differentiation, namely as proliferative mononucleated myoblasts and as post-mitotic multinucleated myotubes following cell fusion. The dose-response curves obtained for myoblasts were strikingly similar to those shown above for MPCs (Fig. 1C), whereas those for myotubes were quite different in that, despite similar EC50s (Table 1), maximum effects were much less severe (Fig. 1D and Table S1). About $70 \%$ of the cells in myotube cultures survived the highest toxic doses versus approximately $20 \%$ in myoblast cultures. The 
percentage of unaffected myotubes may even be underestimated because myotube cultures comprised up to $50 \%$ of nuclei belonging to non-fused cells (Fig. S2), which may still be proliferating myoblasts. Statin toxicity was also evaluated on neural stem cells (NSCs), which are hES-derived precursors with no relevance to the myogenic lineage. NSCs displayed a very distinct pattern of statin-induced toxicity from that of MPCs, with only limited effects and much higher extrapolated EC50s (Fig. 1E and Table 1).

Simvastatin, one of the four statins that exhibited intermediate acute toxicity profile on MPCs and myoblasts, was chosen for subsequent exploration of long-term toxicity using repeateddose exposure.

\section{Development of a simvastatin repeated-dose exposure model on MPCs.}

In order to set-up an experimental model of repeated-dose exposure, MPCs were exposed to simvastatin, renewed every other day in the culture medium, for 17 days (Fig. 2 and Fig. S3). Three different doses of simvastatin were chosen based on the results of the dose-response curve: the highest dose that did not affect cell numbers $(0.4 \mu \mathrm{M})$, the lowest dose that induced a cell loss at plateau level $(2.5 \mu \mathrm{M})$ and an intermediate dose $(1 \mu \mathrm{M})$ (Fig. 2). Toxicity was appreciated on cumulated cell numbers as compared to controls that only received vehicle DMSO. The $2.5 \mu \mathrm{M}$ dose induced a rapid drop in cell numbers, leaving almost no surviving cells after 7 days (Fig. 2A). The $0.4 \mu \mathrm{M}$ dose had no statistically significant effect on cumulated cell number over time although apparent doubling times slightly higher than controls were observed (Fig. 2A-B). In contrast, the $1 \mu \mathrm{M}$ dose resulted in a partial and sustained decrease in cumulated cell number as compared to controls (Fig. 2A), with apparent doubling times significantly higher during the whole experiment (Fig. 2B), therefore unveiling a continued long-term effect of the drug. The $1 \mu \mathrm{M}$ concentration was, therefore, used as an inducer of long-term adverse effects of simvastatin in subsequent experiments. 
The cellular mechanisms leading to the sustained reduction of cumulated MPC number under the $1 \mu \mathrm{M}$ simvastatin treatment were explored by analyzing in parallel cell death and cell proliferation at different time points following repeated-dose exposure (Fig. S3). Once adjusted to the level of basal cell death occurring in control conditions, there was very little, if any, additional cell death under the $1 \mu \mathrm{M}$ treatment (Fig. 2C). This clearly differed from the results obtained after 2 days of exposure with the $2.5 \mu \mathrm{M}$ concentration, which showed a 2.5 fold increase in cell death, signing a direct cytotoxic effect. In contrast to the absence of direct induction of cell lethality, cell proliferation was significantly affected by simvastatin $1 \mu \mathrm{M}$ at all time-points studied (Fig. 2D). Quantification of cells undergoing proliferation using flow cytometry showed a significant dose-dependent decrease in the proportion of proliferating cells after 2 days of exposure to the drug. Repeated exposures to simvastatin $1 \mu \mathrm{M}$ caused a continued cytostatic effect, as monitored after 9 and 16 days of treatment (Fig. 2D).

In order to check the cell-specificity of those simvastatin effects, NSCs were also exposed to the same doses of simvastatin (Fig. S4). Prolonged exposure of NSCs to the $2.5 \mu \mathrm{M}$ highest dose induced a small increase of apparent doubling time during the first week (Fig. S4A), as well as a slight decrease of cell proliferation after 9 days of exposure (Figure S4B). The two lower doses had no significant effect on NSCs.

The cytostatic effect of simvastatin $1 \mu \mathrm{M}$ on MPCs was reversible when the drug was withdrawn from the culture medium after 8 days of exposure (Fig. S5). One day after withdrawal, the MPCs began to proliferate again actively (Fig. S5C). The proportion of replicating cells was even higher than in untreated control cells during the first few days after drug withdrawal, before reaching back control level (Fig. S5C). 


\section{Molecular changes induced by simvastatin repeated-dose exposure in MPCs.}

In order to monitor molecular correlates of the response to simvastatin repeated-dose exposure, we used a set of soluble ligands derived from the receptor-binding domains of retroviral envelope glycoproteins (RBDs), previously described to detect metabolic reprogramming [21-26]. RBDs bind cell surface receptors that belong to the solute carrier (SLC) family, thus allowing quantification of nutrient transporters present at the cell surface. Binding of a set of 9 RBDs was quantified by flow cytometry in MPCs at different time points of the repeated-dose exposure to simvastatin (Fig. S3). RBD flow cytometry data revealed a characteristic nutrient transporter pattern that distinguished prolonged exposures from short-term exposures (Fig. 3A). A trend towards increased RBD binding was measured for most nutrient transporters after 1 day of treatment (D1), except for the glucose transporter Glut1 and inorganic phosphate importer PiT2 (Fig. 3A). Those two transporters were decreased from the beginning of the exposure and were grouped separately from the other nutrient transporters when applying hierarchical clustering. A general decrease of RBD binding to all nutrient transporters as compared to DMSO-treated controls characterized prolonged treatments (D3, D10 and D17). Quantitative RT-PCR analysis of the corresponding SLC genes showed that decrease of RBD binding to PiT2 could be due, at least partly, to transcriptional mechanisms (Fig. 3B). This effect was fully rescued by simultaneous mevalonate treatment (Fig. S6). In contrast, gene expression for PiT1, another inorganic phosphate transporter, and for $X P R l$, a newly described inorganic phosphate exporter [27], did not correlate with changes in RBD binding, suggesting post-translational modulations (Fig. 3C-D).

In order to further explore the differences at the molecular level between single and repeateddose exposures to simvastatin, a whole genome transcriptomic analysis was performed on 
MPCs treated with simvastatin $1 \mu \mathrm{M}$ either for 48 hours with a single dose exposure or with repeated-dose exposure for 17 days (Fig. S3). A massive perturbation in gene expression followed the acute single dose exposure, with a total of 3,740 differentially expressed genes when compared to DMSO treated controls $(|F C| \geq 2$, p < 0.05) (Fig. 4A-B). However, the prolonged drug exposure dramatically decreased the number of differentially expressed genes to 139 after 17 days of treatment (Fig. 4A-B). Of those 139 genes, 54 were already altered after short-term exposure and 85 were specifically modulated after prolonged exposure (Fig. 4B).

After single dose drug exposure, a massive up-regulation was observed in particular for genes implicated in lipid and glycoprotein biosynthesis and/or metabolic processes (Table 2), as well as in lysosomal activity (KEGG enriched process; P-value 3.30E-06, 54 genes). Downregulated genes were mostly related to mitosis and cell cycle (Table 2). These pathways were not significantly altered after 17 days of exposure to the drug (Table 2). Quantitative RT-PCR of representative genes for cholesterol biosynthesis and lipid metabolism (HMGCR, LDLR and the transcription factor $S R E B P F 2$ regulating their expression) confirmed an acute upregulation followed by a complete adaptation over time in the repeated-dose exposure set-up (Fig. S7). After the repeated-dose exposure of 17 days, gene expression changes were mainly related to tissue morphogenesis, tissue development, and regulation of cell differentiation (Table 2). More specifically, negative regulation of muscle development was one of the enriched terms. To refine this pathway enrichment analysis, "Physiological System Development and Function" terms significantly associated with differentially expressed genes were identified using IPA software (Table S2). Repeated-dose exposure of 17 days induced changes in skeletal and muscular system development and function, among which proliferation and migration processes were affected. On the other hand, the acute treatment of 
2 days did not induce any significant change in any of those terms. The expressions of a few genes differentially expressed after repeated-dose exposure and involved in those pathways were validated by RT-qPCR (Table 2 and Fig. S8).

The evolution of $F B X O 32$, which encodes the protein atrogin-1, an E3 ubiquitin ligase involved in skeletal muscle atrophy [28], was also checked by quantitative RT-PCR (Fig. 5A). Atrogin-1 mRNA level increased significantly in response to simvastatin $2.5 \mu \mathrm{M}$, while it was not altered as compared to controls in the acute phase following exposure to simvastatin 1 $\mu \mathrm{M}$. In contrast, repeated-dose exposure to simvastatin $1 \mu \mathrm{M}$ resulted in a $50 \%$ decrease in atrogin-1 gene expression following 10 or 17 days of treatment. This decrease of atrogin-1 expression was dependent of HMGCR inhibition as it was fully rescued by simultaneous mevalonate treatment.

To evaluate a possible functional link between this down-regulation of atrogin-1 and the decreased proliferation of MPCs observed after prolonged exposure to simvastatin, the expression of atrogin-1 was down-regulated by the use of 2 distinct siRNAs in MPCs and primary myoblasts. Cell numbers were subsequently quantified by Hoechst labeling, and proliferation by Edu incorporation, both measured using high content imaging (Fig. S9). 48 hours after siRNA transfection, knock-down of atrogin-1 (Fig. S9A) resulted in a decrease in the proportion of Edu positive cells in both cell types (Fig. S9B). This down-regulation of proliferation resulted in a significant decrease in cell number $72 \mathrm{~h}$ after siRNA transfection for both siRNAs in myoblasts and at least for one siRNA in MPCs (Fig. S9C).

\section{Cerivastatin repeated-dose exposure of MPCs recapitulates simvastatin repeated-dose} effects.

To discriminate between possible specific effects of simvastatin and more general features of statin exposure, repeated-dose exposure of MPCs to another statin was explored. Cerivastatin, 
which displayed the most distinct toxic profile from simvastatin after short-term exposure (Fig. 1A and Table 1), was used for that control. To be able to compare extended exposures, equivalent concentrations to the 3 simvastatin ones were calculated for cerivastatin, using the equations of the $48 \mathrm{~h}$ dose-response curves. The effects of those 3 cerivastatin concentrations$16 \mathrm{nM}, 55 \mathrm{nM}$ and $180 \mathrm{nM}$ respectively- on MPC cumulated cell numbers and apparent doubling times were very similar to those of simvastatin (Fig. S10A-B). The highest dose was significantly cytotoxic after 2 days of exposure whereas, after 9 days, the 2 lower doses, allowing extended exposure, did not induce significant cell death (Fig. S10C). Similarly to simvastatin, a clear cytostatic effect was measured after 2 days of exposure for the 2 higher doses (Fig. S10D). Despite some adaptation, a significant cytostatic effect was still measured after 9 days of exposure to the intermediate dose of $55 \mathrm{nM}$ (Fig. S10D).

At the molecular level, cerivastatin induced deregulations of expression of atrogin-1 (FBXO32), KLF4, BMP4 and ACAN, which were very similar to those observed after simvastatin treatment, for both short-term and long-term exposures (Fig S10E). 


\section{Discussion}

The main result of this study is the demonstration that repeated-dose exposure to simvastatin may elicit an adverse cytostatic effect on cells of the myogenic lineage in the absence of cell death. This long-term effect was associated with molecular changes that strongly differed from those induced by acute exposure of cells to the same dose of the drug, thus revealing powerful adaptive mechanisms. Cerivastatin treatment provoked similar changes, suggesting that these observations are not specific to simvastatin but may correspond more broadly to statin effects. These results were obtained using derivatives of human pluripotent stem cells, thus demonstrating their value as human cellular resources for implementing in vitro repeated-dose toxicity studies.

\section{Repeated-dose exposure to simvastatin may induce myotoxicity through a cytostatic} effect in the absence of cell death.

The present results demonstrate that extended treatment with statins may have profound deleterious functional effects in the absence of cell death, by inhibiting cell proliferation. This mechanism had been suggested by van Vliet et al. [20] on the basis of the analysis of human myoblast cultures, but the use of a single drug exposure paradigm did not allow the authors to draw a firm conclusion. Instead, most studies have subsequently emphasized the existence of massive cell death of muscle cells following in vitro treatment $[6,7,9,11-14]$. Massive and rapid cell death were also observed here, when the dosage of simvastatin was increased from 1 to $2.5 \mu \mathrm{M}$, indicating the existence of a dose-dependent sequence of deleterious effects of the drug, the inhibition of cell proliferation occurring independently of cell death at a lower dosage. Extrapolation of drug dosages from the in vitro to the clinical situation is difficult, in particular because only plasma titers of the drug are available in patients, and tissue concentrations may strongly differ. Drug doses used in in vitro studies reporting cell death 
were often far higher than the usual therapeutic dosages, ranging for simvastatin between 60 and 1000 times the peak plasmatic titer recorded in patients $[6-8,11,13,29,30]$. Despite still being above therapeutic doses, the $1 \mu \mathrm{M}$ intermediate dose used here to induce the long-term cytostatic effect ranges approximately between 10 and 40 times the peak plasma titer in patients [31], overall lower than the doses previously shown to induce cell death and/or apoptosis.

The possibility that inhibition of proliferation may contribute to myotoxicity is consistent with well-known mechanisms of muscle regeneration. Muscle injury normally activates a muscle repair process that involves satellite cells activation, proliferation, differentiation and fusion to the damaged myofibers [32]. If muscle progenitors are not able to proliferate properly because of the effects of statins, neither muscle repair nor regeneration of a pool of satellite cells would occur normally. Intense physical exercise is known to induce muscle injury requiring active regeneration and this has been repeatedly underlined as a major cause for susceptibility to statin myotoxicity $[33,34]$. Some treated patients experimented muscular symptoms after an intense physical exercise [35-37]. Strikingly, only $20 \%$ of a cohort of top athletes needing statin medication tolerated the treatment without muscular side effects, a much higher proportion than in the general population [38].

This hypothesis of a muscle repair process impaired by a defective proliferation is strengthened by studies that describe, in addition, an inhibition of the fusion of myoblasts into myotubes by simvastatin $1 \mu \mathrm{M}$ in vitro and in vivo [10] and by other statins in vitro [39]. Interestingly, simvastatin repeated-dose treatment resulted in our model in an up-regulation of genes involved in tissue morphogenesis, tissue development, cell differentiation, and more specifically, in negative regulation of muscle development (Table 2), suggesting phenotypic changes that might also impact muscle repair. Blockade of proliferation as a main cause of statin-induced myotoxicity rather than- or in addition to- muscle cell death would also fit well 
with the clinical observation of the very rapid curative effect of drug withdrawal $[38,40,41]$. Accordingly, the cytostatic effect of simvastatin $1 \mu \mathrm{M}$ was immediately reversible upon withdrawal of the drug in the present study. The hypothesis that statins may have antiproliferative effects on muscle cells is also strengthened indirectly by data that concern other cell types. Such an effect has in particular been observed on vascular smooth muscle cells at doses close to therapeutic ones $[42,43]$, an effect which has been deemed to contribute to their therapeutic action on the cardiovascular system.

\section{Adaptive mechanisms contribute to cell responses to repeated-dose toxicity.}

The comparative analyses of molecular changes induced by simvastatin $1 \mu \mathrm{M}$ treatment after single and repeated-dose exposures revealed major differences. Among them, it is interesting to mention that single drug exposure resulted in up-regulation of sterol and lipid metabolic pathways, while there was no alteration registered anymore after 17 days of exposure. In response to intracellular cholesterol depletion, expression of enzymes of the cholesterol biogenesis pathway are up-regulated in part via the activation of the transcription factor SREBPF2 [44, 45], which is also able to activate its own transcription [46]. Intracellular cholesterol depletion induced in vitro by statin treatment results in transcriptional upregulation of $H M G C R$ and/or of low density lipoprotein receptor (LDLR) [47-50]. SREBPF2, $H M G C R$ and $L D L R$ were indeed up-regulated in the present study. Since the experiments were performed with culture medium not depleted from cholesterol, transient up-regulation of $L D L R$ may have been sufficient to allow exogenous cholesterol to enter the cells and restore normal levels of the enzymes of the cholesterol pathway despite ongoing simvastatin treatment. Interestingly, despite a cholesterol metabolism pathway returning back to normal, the expression of several molecular markers -some of which indicative of phenotypic 
changes, such as $A C A N, B M P 4$ or $K L F 4$ (Fig. S7)- remained or were specifically induced by the repeated-dose exposure to simvastatin.

The gene encoding atrogin-1 attracted our attention because of its implication in skeletal muscle atrophy [28]. Changes in expression of this E3 ubiquitin ligase have been observed in atrophic muscle following spinal cord injury, being up-regulated in the acute phase [51] and down-regulated during the chronic stage [52]. Results obtained in the present study bear some resemblance to this dual response. Indeed, atrogin-1 transcript was significantly up-regulated after single exposure to the cytotoxic $2.5 \mu \mathrm{M}$ dose, as in previous studies of statin effects [9, 53]. In sharp contrast, atrogin-1 was significantly down-regulated following repeated-dose exposure. Our results thus suggest a specific sequence of atrogin-1 expression changes associated to acute versus chronic myotoxicity. Besides, siRNAs targeting atrogin-1 expression induced a decrease in the proliferation rate of MPCs and primary myoblasts. Although a comprehensive analysis of molecular changes that may impact proliferation after prolonged simvastatin treatment was beyond the scope of this study, down-regulation of atrogin-1 may thus contribute to the cytostatic effect measured in our model.

The metabolism of MPCs was also differentially impacted by single versus repeated simvastatin treatment, as revealed by the distinct nutrient transporter expression patterns observed in MPCs following short-term or prolonged exposures. Nutrient transporters are key players of cell homeostasis and play a pivotal role in metabolic adaptation to environmental changes $[24,26,54]$. Differences in their expression have already been described under perturbed conditions $[19,21,22]$. In the present study, all nutrient transporters indeed demonstrated altered expression at one point or another of the repeated-dose treatment. Most -with the exception of Glut1 and PiT2- were actually specifically down-regulated after prolonged exposure to the drug as compared to the short-term exposure (day 1). This result 
further emphasizes the differences between immediate and protracted responses of MPCs to simvastatin treatment.

Altogether, the results of all the molecular analyses performed here point to major mechanistic differences between cell responses to short-term versus prolonged statin exposures and indicate the triggering of adaptive mechanisms that are accompanying the development of long-term toxicity.

Derivatives of human pluripotent stem cells show unique advantages for the in vitro evaluation of repeated-dose drug toxicity.

The present model was based on derivatives of human pluripotent stem cells. Implementation of in vitro repeated-dose exposure models has faced the challenge of maintaining cells exposed to treatment for extended periods of time [55]. The majority of available human cellbased models rely on the use of either primary cells isolated from human tissues or immortalized cell lines, both presenting several limitations for the development of repeateddose exposure models. Maintaining primary cell cultures for extended periods of time is especially difficult because of their limited lifespan. Besides, human tissue material needed to establish primary cultures is a biological resource of limited access. Immortalized cell lines provide an alternative to address both issues. However, they have been genetically modified to express a tumor-related or viral gene to gain their "immortal" status, thus often deviating phenotypically, genetically and functionally from their original counterparts.

Contrary to human primary or immortalized cells, human pluripotent stem cells can be expanded indefinitely in culture while keeping normal karyotypes [56, 57]. Under appropriate culture conditions, they can be differentiated theoretically into any cell type of the organism, thus providing the opportunity to develop cell-based models to study acute and repeated-dose 
exposure toxicities on different tissues of interest, including those difficult to access through primary cells [58].

However, the relevance of MPCs derived from hES cells for studying the effects of statins on adult muscles can be questioned. MPCs are not fully differentiated myogenic cells, even if a sub-population has been shown by some authors to fuse to form myotubes in vitro, and with muscle fibers in vivo following transplantation [17]. It was encouraging that hES-derived MPCs displayed very similar statin-induced acute toxicity patterns as compared to primary human myoblasts, and that these effects were specific to the myogenic lineage since hESderived NSCs did not exhibit a similar response. The use of MPCs allowed to demonstrate that cellular responses to short-term or prolonged statin treatment were quantitatively and qualitatively different and to draw new mechanistic hypotheses. 


\section{Summary}

In this study, human embryonic stem cell-derived mesodermal precursors of the myogenic lineage were used to implement and characterize simvastatin repeated-dose exposure effects in vitro. Prolonged exposures to simvastatin resulted in very distinct responses as compared to short-term exposure, both at the functional and molecular levels. Comparative analysis of molecular modifications induced by simvastatin short-term versus prolonged exposures demonstrated a major adaptive cell response characterized by phenotypic changes and metabolic reprogramming. At the functional level, simvastatin extended exposure resulted mainly in a loss of cell proliferative capacity in the absence of concomitant cell death. We propose a new mechanistic hypothesis regarding statin-induced myotoxicity, pointing to inhibition of the proliferation of muscle precursor cells -that would prevent proper muscle regeneration- as a major contributor to chronic myotoxicity. This study demonstrates the need for more models based on long-term drug exposures to study chronic toxicities. Derivatives of human pluripotent stem cells appear as a nicely suited human cell resource for such studies. 
Acknowledgements: This study has been carried out within the FP7 EC funded network SCR\&Tox (Grant Agreement no. 266753) and was also supported in part by a EC funded Marie Curie Career Integration Grant (no. 322283). I-Stem is part of the Biotherapies Institute for Rare Diseases (BIRD) supported by the Association Française contre les Myopathies (AFM-Téléthon).

Disclosure of Potential Conflicts of Interest: Christelle Cousin and Vincent Petit are employees of the company Metafora biosystems which develops biomarkers based on metabolite transporters in physiological and pathological conditions. Marc Sitbon is a cofounder of Metafora biosystems. 


\section{References}

1 Mihos CG, Pineda AM, Santana O. Cardiovascular effects of statins, beyond lipidlowering properties. Pharmacological research : the official journal of the Italian Pharmacological Society. 2014.

2 Corsini A, Maggi FM, Catapano AL. Pharmacology of competitive inhibitors of HMG-CoA reductase. Pharmacological research : the official journal of the Italian Pharmacological Society. 1995;31:9-27.

3 Thompson PD, Clarkson P, Karas RH. Statin-associated myopathy. JAMA : the journal of the American Medical Association. 2003;289:1681-1690.

4 Furberg CD, Pitt B. Withdrawal of cerivastatin from the world market. Current controlled trials in cardiovascular medicine. 2001;2:205-207.

5 Vaklavas C, Chatzizisis YS, Ziakas A et al. Molecular basis of statin-associated myopathy. Atherosclerosis. 2009;202:18-28.

6 Kwak HB, Thalacker-Mercer A, Anderson EJ et al. Simvastatin impairs ADPstimulated respiration and increases mitochondrial oxidative stress in primary human skeletal myotubes. Free radical biology \& medicine. 2012;52:198-207.

7 Sacher J, Weigl L, Werner M et al. Delineation of myotoxicity induced by 3-hydroxy3-methylglutaryl CoA reductase inhibitors in human skeletal muscle cells. The Journal of pharmacology and experimental therapeutics. 2005;314:1032-1041.

8 Nishimoto T, Tozawa R, Amano Y et al. Comparing myotoxic effects of squalene synthase inhibitor, T-91485, and 3-hydroxy-3-methylglutaryl coenzyme A (HMG$\mathrm{CoA}$ ) reductase inhibitors in human myocytes. Biochemical pharmacology. 2003;66:2133-2139. 
9 Hanai J, Cao P, Tanksale $\mathrm{P}$ et al. The muscle-specific ubiquitin ligase atrogin1/MAFbx mediates statin-induced muscle toxicity. The Journal of clinical investigation. 2007;117:3940-3951.

10 Trapani L, Segatto M, La Rosa P et al. 3-hydroxy 3-methylglutaryl coenzyme A reductase inhibition impairs muscle regeneration. Journal of cellular biochemistry. 2012;113:2057-2063.

11 Masters BA, Palmoski MJ, Flint OP et al. In vitro myotoxicity of the 3-hydroxy-3methylglutaryl coenzyme A reductase inhibitors, pravastatin, lovastatin, and simvastatin, using neonatal rat skeletal myocytes. Toxicology and applied pharmacology. 1995;131:163-174.

12 Sakamoto K, Honda T, Yokoya S et al. Rab-small GTPases are involved in fluvastatin and pravastatin-induced vacuolation in rat skeletal myofibers. FASEB journal : official publication of the Federation of American Societies for Experimental Biology. 2007;21:4087-4094.

13 Itagaki M, Takaguri A, Kano S et al. Possible mechanisms underlying statin-induced skeletal muscle toxicity in L6 fibroblasts and in rats. Journal of pharmacological sciences. 2009;109:94-101.

14 Johnson TE, Zhang X, Bleicher KB et al. Statins induce apoptosis in rat and human myotube cultures by inhibiting protein geranylgeranylation but not ubiquinone. Toxicology and applied pharmacology. 2004;200:237-250.

15 Mullen PJ, Luscher B, Scharnagl H et al. Effect of simvastatin on cholesterol metabolism in $\mathrm{C} 2 \mathrm{C} 12$ myotubes and HepG2 cells, and consequences for statininduced myopathy. Biochemical pharmacology. 2010;79:1200-1209. 
16 Sakamoto K, Mikami H, Kimura J. Involvement of organic anion transporting polypeptides in the toxicity of hydrophilic pravastatin and lipophilic fluvastatin in rat skeletal myofibres. British journal of pharmacology. 2008;154:1482-1490.

17 Barberi T, Bradbury M, Dincer Z et al. Derivation of engraftable skeletal myoblasts from human embryonic stem cells. Nature medicine. 2007;13:642-648.

18 Giraud-Triboult K, Rochon-Beaucourt C, Nissan X et al. Combined mRNA and microRNA profiling reveals that miR-148a and miR-20b control human mesenchymal stem cell phenotype via EPAS1. Physiological genomics. 2011;43:77-86.

19 Li J, Cousin C, Tinkler H et al. Profiling of Nutrient Transporter Expression in Human Stem Cell-Derived Cardiomyocytes Exposed to Tyrosine Kinase Inhibitor Anticancer Drugs Using RBD Ligands. Journal of biomolecular screening. 2014.

20 van Vliet AK, Negre-Aminou P, van Thiel GC et al. Action of lovastatin, simvastatin, and pravastatin on sterol synthesis and their antiproliferative effect in cultured myoblasts from human striated muscle. Biochemical pharmacology. 1996;52:13871392.

21 Lagrue E, Abe H, Lavanya M et al. Regional characterization of energy metabolism in the brain of normal and MPTP-intoxicated mice using new markers of glucose and phosphate transport. Journal of biomedical science. 2010;17:91.

22 Laval J, Touhami J, Herzenberg LA et al. Metabolic adaptation of neutrophils in cystic fibrosis airways involves distinct shifts in nutrient transporter expression. Journal of immunology. 2013;190:6043-6050.

23 Manel N, Kim FJ, Kinet $\mathrm{S}$ et al. The ubiquitous glucose transporter GLUT-1 is a receptor for HTLV. Cell. 2003;115:449-459. 
24 Montel-Hagen A, Blanc L, Boyer-Clavel M et al. The Glut1 and Glut4 glucose transporters are differentially expressed during perinatal and postnatal erythropoiesis. Blood. 2008;112:4729-4738.

25 Oburoglu L, Tardito S, Fritz V et al. Glucose and Glutamine Metabolism Regulate Human Hematopoietic Stem Cell Lineage Specification. Cell stem cell. 2014.

26 Swainson L, Kinet S, Manel N et al. Glucose transporter 1 expression identifies a population of cycling CD4+ CD8+ human thymocytes with high CXCR4-induced chemotaxis. Proceedings of the National Academy of Sciences of the United States of America. 2005;102:12867-12872.

27 Giovannini D, Touhami J, Charnet P et al. Inorganic phosphate export by the retrovirus receptor XPR1 in metazoans. Cell reports. 2013;3:1866-1873.

28 Gomes MD, Lecker SH, Jagoe RT et al. Atrogin-1, a muscle-specific F-box protein highly expressed during muscle atrophy. Proceedings of the National Academy of Sciences of the United States of America. 2001;98:14440-14445.

29 Tanaka S, Sakamoto K, Yamamoto M et al. Mechanism of statin-induced contractile dysfunction in rat cultured skeletal myofibers. Journal of pharmacological sciences. 2010;114:454-463.

30 Vaughan RA, Garcia-Smith R, Bisoffi M et al. Ubiquinol rescues simvastatinsuppression of mitochondrial content, function and metabolism: implications for statin-induced rhabdomyolysis. European journal of pharmacology. 2013;711:1-9.

31 Bellosta S, Paoletti R, Corsini A. Safety of statins: focus on clinical pharmacokinetics and drug interactions. Circulation. 2004;109:III50-57.

32 Tedesco FS, Dellavalle A, Diaz-Manera J et al. Repairing skeletal muscle: regenerative potential of skeletal muscle stem cells. The Journal of clinical investigation. 2010;120:11-19. 
33 Thompson PD, Zmuda JM, Domalik LJ et al. Lovastatin increases exercise-induced skeletal muscle injury. Metabolism: clinical and experimental. 1997;46:1206-1210.

34 Seachrist JL, Loi CM, Evans MG et al. Roles of exercise and pharmacokinetics in cerivastatin-induced skeletal muscle toxicity. Toxicological sciences : an official journal of the Society of Toxicology. 2005;88:551-561.

35 Chrysanthopoulos C, Kounis N. Rhabdomyolysis due to combined treatment with lovastatin and cholestyramine. Bmj. 1992;304:1225.

36 Sinzinger H, Schmid P, O'Grady J. Two different types of exercise-induced muscle pain without myopathy and CK-elevation during HMG-Co-enzyme-A-reductase inhibitor treatment. Atherosclerosis. 1999;143:459-460.

37 Unnikrishnan D, Satish B. Exertion-induced rhabdomyolysis in a patient on statin therapy. Nephrology, dialysis, transplantation : official publication of the European Dialysis and Transplant Association - European Renal Association. 2005;20:244.

38 Sinzinger H, O'Grady J. Professional athletes suffering from familial hypercholesterolaemia rarely tolerate statin treatment because of muscular problems. British journal of clinical pharmacology. 2004;57:525-528.

39 Belo RS, Jamieson JC, Wright JA. Studies on the effect of mevinolin (lovastatin) and mevastatin (compactin) on the fusion of L6 myoblasts. Molecular and cellular biochemistry. 1993;126:159-167.

40 Soininen K, Niemi M, Kilkki E et al. Muscle symptoms associated with statins: a series of twenty patients. Basic \& clinical pharmacology \& toxicology. 2006;98:51-54.

41 Tomlinson SS, Mangione KK. Potential adverse effects of statins on muscle. Physical therapy. 2005;85:459-465.

42 Chan KC, Wang CJ, Ho HH et al. Simvastatin inhibits cell cycle progression in glucose-stimulated proliferation of aortic vascular smooth muscle cells by up- 
regulating cyclin dependent kinase inhibitors and p53. Pharmacological research : the official journal of the Italian Pharmacological Society. 2008;58:247-256.

43 Porter KE, Naik J, Turner NA et al. Simvastatin inhibits human saphenous vein neointima formation via inhibition of smooth muscle cell proliferation and migration. Journal of vascular surgery. 2002;36:150-157.

44 Brown MS, Goldstein JL. The SREBP pathway: regulation of cholesterol metabolism by proteolysis of a membrane-bound transcription factor. Cell. 1997;89:331-340.

45 Horton JD, Shah NA, Warrington JA et al. Combined analysis of oligonucleotide microarray data from transgenic and knockout mice identifies direct SREBP target genes. Proceedings of the National Academy of Sciences of the United States of America. 2003;100:12027-12032.

46 Sato R, Inoue J, Kawabe Y et al. Sterol-dependent transcriptional regulation of sterol regulatory element-binding protein-2. The Journal of biological chemistry. $1996 ; 271: 26461-26464$.

47 Mangravite LM, Engelhardt BE, Medina MW et al. A statin-dependent QTL for GATM expression is associated with statin-induced myopathy. Nature. 2013;502:377380.

48 Medina MW, Gao F, Naidoo D et al. Coordinately regulated alternative splicing of genes involved in cholesterol biosynthesis and uptake. PloS one. 2011;6:e19420.

49 Stormo C, Kringen MK, Grimholt RM et al. A novel 3-hydroxy-3-methylglutarylcoenzyme A reductase (HMGCR) splice variant with an alternative exon 1 potentially encoding an extended N-terminus. BMC molecular biology. 2012;13:29.

50 Yamasaki D, Nakamura T, Okamura N et al. Effects of acid and lactone forms of 3hydroxy-3-methylglutaryl coenzyme A reductase inhibitors on the induction of MDR1 expression and function in LS180 cells. European journal of pharmaceutical sciences : 
official journal of the European Federation for Pharmaceutical Sciences. 2009;37:126132.

51 Urso ML, Chen YW, Scrimgeour AG et al. Alterations in mRNA expression and protein products following spinal cord injury in humans. The Journal of physiology. 2007;579:877-892.

52 Leger B, Senese R, Al-Khodairy AW et al. Atrogin-1, MuRF1, and FoXO, as well as phosphorylated GSK-3beta and 4E-BP1 are reduced in skeletal muscle of chronic spinal cord-injured patients. Muscle \& nerve. 2009;40:69-78.

53 Mallinson JE, Constantin-Teodosiu D, Sidaway J et al. Blunted Akt/FOXO signalling and activation of genes controlling atrophy and fuel use in statin myopathy. The Journal of physiology. 2009;587:219-230.

54 Beck L, Leroy C, Beck-Cormier S et al. The phosphate transporter PiT1 (Slc20a1) revealed as a new essential gene for mouse liver development. PloS one. 2010;5:e9148.

55 Prieto P, Baird AW, Blaauboer BJ et al. The assessment of repeated dose toxicity in vitro: a proposed approach. The report and recommendations of ECVAM workshop 56. Alternatives to laboratory animals : ATLA. 2006;34:315-341.

56 Thomson JA, Itskovitz-Eldor J, Shapiro SS et al. Embryonic stem cell lines derived from human blastocysts. Science. 1998;282:1145-1147.

57 Takahashi K, Tanabe K, Ohnuki M et al. Induction of pluripotent stem cells from adult human fibroblasts by defined factors. Cell. 2007;131:861-872.

58 Wobus AM, Loser P. Present state and future perspectives of using pluripotent stem cells in toxicology research. Archives of toxicology. 2011;85:79-117. 
59. Feyeux M, Bourgois-Rocha F, Redfern A et al. Early transcriptional changes linked to naturally occurring Huntington's disease mutations in neural derivatives of human embryonic stem cells. Human molecular genetics. 2012;21:3883-3895.

60. Sebe P, Doucet C, Cornu JN et al. Intrasphincteric injections of autologous muscular cells in women with refractory stress urinary incontinence: a prospective study. International urogynecology journal. 2011;22:183-189. 


\section{Figure Legends}

Figure 1. Dose-response curves of the toxicity of statins following single-dose exposure. hES-derived MPCs in absence (A) or presence (B) of $200 \mu \mathrm{M}$ mevalonate, primary myoblasts (C), myotubes differentiated from primary myoblasts (D), and hES-derived NSCs (E), were treated in 384-well plates with the indicated statins for $48 \mathrm{~h}$. Cell nuclei were quantified by high content imaging using Hoechst staining. Results of residual nuclei number are expressed as means \pm SD in statin-treated cells normalized to DMSO treated cells $(n=4$ for myotubes, $n$ $=3$ for other cell types).

\section{Figure 2. Characterization of simvastatin-induced acute and repeated-dose exposure} responses in MPCs

(A) Cumulated cell numbers of MPCs treated with repeated doses of the indicated concentrations of simvastatin or vehicle DMSO during 14 days. Data are presented as means $\pm \mathrm{SD}(\mathrm{n}=4)$. (B) Average apparent doubling times during the first and second weeks of treatments calculated according to the cell counts (means $\pm \mathrm{SD}, \mathrm{n}=4$ ). ${ }^{* *} \mathrm{p}<0.01, * * * \mathrm{p}<0.001$ determined by ANOVA followed by Dunnett's comparison to DMSO condition. (C) Cell mortality analysis of MPCs treated with simvastatin. MPCs were labeled with Annexin VFITC and propidium iodide and analyzed by flow cytometry. The total of early apoptotic and dead cells was quantified as Annexin-V+ cells (red gates). Left panel shows representative data obtained on day 2 of treatment. Right panel shows mortality quantifications obtained on the indicated days of repeated-dose exposure. (D) Proliferation rate analysis of MPCs treated with simvastatin and evaluated by Edu incorporation quantification by flow cytometry. Left panel shows representative data obtained on day 2 of treatment and Edu positive gating. Right panel shows Edu quantifications obtained on the indicated days of repeated-dose exposure. 
(C, D) Results have been normalized to DMSO treated cells and are expressed as means \pm SD $(\mathrm{n}=4) . \dagger$ not determined because of cell death; ${ }^{*} \mathrm{p}<0.05,{ }^{*} \mathrm{p}<0.01,{ }^{* * *} \mathrm{p}<0.001$ determined by one sample t test.

Figure 3. Expression profiling of nutrient transporters at the surface of MPCs in response to simvastatin repeated-dose exposure

(A) Unsupervised hierarchical clustering of quantifications of nutrient transporters at the cell surface of MPCs treated with repeated doses of simvastatin. Nutrient transporters were quantified by flow cytometry using RBDs after 1, 3, 10 or 17 days of treatment (D1, D3, D10 and D17) except for the $2.5 \mu \mathrm{M}$ dose, which could be analyzed only after one day (D1) of treatment. Experiments were performed in duplicates (indicated as \#1 and \#2) and data in the cluster are presented as the results of a single experiment normalized to DMSO. (B, C, D) Relative cell surface expression using RBD technology (left panels) and mRNA levels quantified by qRT-PCR (right panels) of the transporters PiT2 (B), PiT1 (C), and XPR1 (D) in MPCs treated with repeated doses of simvastatin or vehicle DMSO. Cell surface expression results are expressed as means $\pm \mathrm{SD}(\mathrm{n}=3)$ of the expression in simvastatin-treated cells normalized to the expression in DMSO-treated cells. Relative mRNA levels are expressed as means \pm SD $(n=3)$ of transcript expression in treated cells (DMSO or simvastatin) normalized to the expression in non treated cells. $* \mathrm{p}<0.05, * * \mathrm{p}<0.01, * * * \mathrm{p}<0.001$ determined by one sample $\mathrm{t}$ test $(\mathrm{B}, \mathrm{C}, \mathrm{D}$, left panels) or ANOVA followed by Dunnett's comparison test to $\mathrm{DMSO}(\mathrm{B}, \mathrm{C}, \mathrm{D}$, right panels).

Figure 4. Differentially expressed genes in MPCs in response to simvastatin $1 \mu \mathrm{M}$ repeated-dose exposure. 
(A) Number of differentially expressed genes following treatment with simvastatin $1 \mu \mathrm{M}$ as compared to DMSO ( $|\mathrm{FC}| \geq 2$; $<<0.05$ ). (B) Venn diagram comparing number and distribution of differentially expressed genes following treatment with simvastatin $1 \mu \mathrm{M}$ during 2 and 17 days respectively. (B) Heatmap representing color-coded hierarchical clustering of FC expression values from microarray analysis, after 2 days or 17 days of simvastatin $1 \mu \mathrm{M}$ treatment, for the list of genes significantly modulated by the repeated-dose exposure of 17 days (Unpaired 2-sided student's t-test analysis of microarray data, $|\mathrm{FC}| \geq 2$, $\mathrm{p}<0.05, \mathrm{n}=3$ ). In red, genes validated by qRT-PCR analysis (Figure S7).

\section{Figure 5. Atrogin-1 expression in response to simvastatin repeated-dose treatment.}

Atrogin-1 relative mRNA levels quantified by qRT-PCR at different time points in MPCs treated up to 17 days with repeated doses of the indicated concentrations of simvastatin or vehicle DMSO without $(\mathrm{A})$ or with $(\mathrm{B})$ rescuing mevalonate $(200 \mu \mathrm{M})$. Results are expressed as means \pm SD $(n=3)$ of the expression in treated cells (DMSO or simvastatin) normalized to the expression in non treated cells. ${ }^{*} \mathrm{p}<0.05, * * \mathrm{p}<0.01, * * * \mathrm{p}<0.001$ determined by ANOVA followed by Dunnett's comparison test to DMSO. 